\title{
THz extended spectrum of the monodeuterated methyl formate $\left(\mathrm{DCOOCH}_{3}\right)^{\star}$
}

\author{
C. Duan ${ }^{1}$, M. Carvajal ${ }^{2}$, S. Yu ${ }^{3}$, J. C. Pearson ${ }^{3}$, B. J. Drouin ${ }^{3}$, and I. Kleiner ${ }^{4}$ \\ ${ }^{1}$ College of Physical Science and Technology, Central China Normal University, Luoyu Road 152, 430079 Wuhan, PR China \\ 2 Departamento de Física Aplicada, Unidad Asociada CSIC, Facultad de Ciencias Experimentales, Universidad de Huelva, \\ 21071 Huelva, Spain \\ e-mail: miguel.carvajal@dfa.uhu.es \\ 3 Jet Propulsion Laboratory - NASA, California Institute of Technology, 4800, Oak Grove Drive, Pasadena, CA 91109-8099, USA \\ ${ }^{4}$ Laboratoire Interuniversitaire des Systèmes Atmosphériques, UMR CNRS 7583, Université Paris-Est Créteil et Université Paris \\ Diderot, 61 Av. Charles de Gaulle, 94010 Créteil Cedex, France
}

Received 13 November 2014 / Accepted 21 January 2015

\section{ABSTRACT}

\begin{abstract}
Context. Laboratory spectral recordings and an accurate molecular spectral analysis of any potential interstellar molecule are essential for generating a complete spectroscopic line list. This permits predicting the frequencies and intensities of any transition so that subsequently, it can be identified in the interstellar medium.

Aims. Our analysis of $\mathrm{DCOOCH}_{3}$ aims to provide a comprehensive spectral catalog that encompasses as much as possible the frequency coverage of the new-generation far-IR and submillimeter wave observation facilities.

Methods. We newly measured the rotational spectrum of $\mathrm{DCOOCH}_{3}$ in the laboratory of the Jet Propulsion Laboratory in the frequency range of 0.85 to $1.5 \mathrm{THz}$. We jointly analyzed the new data with literature data using the rho axis method, which is a tool developed for the spectral analysis of molecules with large-amplitude internal $\mathrm{CH}_{3}$ rotors.

Results. We fit 27 spectroscopic constants of $\mathrm{DCOOCH}_{3}$ to 3763 transitions with highest values of $J=69$ and $K_{a}=36$ of the ground torsional state with a standard (unitless) deviation of 0.97 . With respect to previous work, this is a significantly better result that was obtained with 2060 more transitions, and we also achieved a better accuracy for the new parameter values.
\end{abstract}

Key words. molecular data - methods: laboratory: molecular - methods: data analysis - ISM: molecules - submillimeter: ISM

\section{Introduction}

The current understanding of interstellar chemistry has required a sustained methodical procedure based on individual isotope specific molecular detections. The steps include the molecular identification, a molecular abundance estimate, and the determination of their physical conditions (Shaw 2006). The more spectral lines of the most abundant interstellar molecules are identified, the more possible the detection of new molecular species becomes. Solid detection can only be achieved through comprehensive laboratory studies of the molecular species, which involves new measurements, spectral analysis, and fits using appropriate Hamiltonians. These detections then enable ever more sophisticated chemical modeling of the interstellar material. Advances in telescope technology continue to extend the limits of spectral databases and demonstrate the need to record and analyze higher frequency spectra of the main insterstellar species and their minor isotopologs.

Methyl formate is a relatively abundant organic molecule in the interstellar medium (ISM). Since its discovery in Sgr B2 (Brown et al. 1975; Churchwell et al. 1975), extensive spectroscopic studies have been carried out for the main species (Bauder 1979; Demaison et al. 1983; Plummer et al. 1984, 1986; Oesterling et al. 1999; Karakawa et al. 2001; Ogata et al. 2004; Carvajal et al. 2007; Maeda et al. 2008b; Ilyushin et al. 2009;

* Full Table A.1 is only available at the CDS via anonymous ftp to cdsarc.u-strasbg.fr (130.79.128.5) or via

http://cdsarc.u-strasbg.fr/viz-bin/qcat?]/A+A/576/A39
Tudorie et al. 2012; Kobayashi et al. 2013) and for its isotopologs (Curl 1959; Oesterling et al. 1995; Willaert et al. 2006; Maeda et al. 2008a,b; Margulès et al. 2009, 2010; Carvajal et al. 2009, 2010; Coudert et al. 2012, 2013; Tercero et al. 2012; Haykal et al. 2014). This spectroscopic database for the methyl formate molecule has given rise to an increasing identification of the spectral lines in different interstellar surveys. In parallel, the new astrophysical observatories (Herschel, ALMA) have generated interest in higher frequency spectral ranges.

In the recent years, a number of molecular line observations toward Orion KL and W51 e2 have been carried out in the ground $v_{\mathrm{t}}=0$, first $v_{\mathrm{t}}=1$ and second $v_{\mathrm{t}}=2$ excited torsional states of main isotopolog (Kobayashi et al. 2007; Demyk et al. 2008; Favre et al. 2011; Takano et al. 2012), toward Orion KL, Sgr B2, NGC 6334 IRS 1, W51 e2, and G19.61-023 in the ground and first excited torsional states of the ${ }^{13} \mathrm{C}$ isotopic substituted species (Carvajal et al. 2009; Haykal et al. 2014; Favre et al. 2014), and toward Orion in the ground state of ${ }^{18} \mathrm{O}$ species (Tercero et al. 2012).

The deuterated species of methyl formate were also concerned, with a number of lines detected for $\mathrm{HCOOCH}_{2} \mathrm{D}$ (Coudert et al. 2013) and a tentative detection in the ground state of the monodeuterated species $\mathrm{DCOOCH}_{3}$ (Margulès et al. 2010). $\mathrm{HCOOCH}_{2} \mathrm{D}$ and $\mathrm{DCOOCH}_{3}$ were identified in the Orion KL nebula with the IRAM 30m telescope. $\mathrm{HCOOCH}_{2} \mathrm{D}$ was detected based on the observation of 66 unblended lines as well as 56 moderately blended lines. For $\mathrm{DCOOCH}_{3}$ the detection was based on more than 100 spectral features with low 
blending effects. Another 300 features that would appear in the survey were heavily blended. We note that the hit-or-miss nature of line identification in congested interstellar survey spectra places a strong requirement on the accuracy and completeness of the spectral line databases. The derived source-averaged total column density for $\mathrm{HCOOCH}_{2} \mathrm{D}$ is $5.0 \times 10^{14} \mathrm{~cm}^{-2}$ and the $\mathrm{HCOOCH}_{2} \mathrm{D} / \mathrm{HCOOCH}_{3}$ column density ratio is about 0.04 in Orion (Coudert et al. 2013). The total column density for $\mathrm{DCOOCH}_{3}$ is $7.8 \times 10^{14} \mathrm{~cm}^{-2}$, and the $\mathrm{DCOOCH}_{3} / \mathrm{HCOOCH}_{3}$ column density ratio varies between 0.02 and 0.06 in the different cloud components of Orion (Margulès et al. 2010). The derived results of the two monodeuterated isotopologs of methyl formate detected in Orion are consistent with each other. The main goal of our present study is to increase the spectroscopic database for $\mathrm{DCOOCH}_{3}$ by measuring new laboratory data and providing a more complete line-list for this species. Data like this are aimed to derive the ISM deuteration of methyl formate with more accuracy and to resolve any anomalous $\mathrm{D} / \mathrm{H}$ ratios that could be observed. Because the $\mathrm{D} / \mathrm{H}$ ratio provides some clues to the molecular formation history, and because this mechanism is not yet understood for methyl formate (Horn et al. 2004), we expect that this study will help to constrain its formation mechanisms in the gas phase and in the icy surfaces and will shed some light on their moments of formation (Taquet et al. 2014).

The microwave spectral range for $\mathrm{DCOOCH}_{3}$ was first recorded between $9-30 \mathrm{GHz}$ by Curl (1959). The spectral recording was then extended to the millimeter- and submillimeter-wave region up to a frequency of $377 \mathrm{GHz}$ by Oesterling et al. (1995). A total of 912 lines up to $J=30$ and $K_{a}=25$ in the ground state combined with the previous reported data were analyzed with a model that Oesterling et al. (1995) named the "global internal axis method" (IAM). More recently, a new spectral recording was carried out up to $661 \mathrm{GHz}$ (Margulès et al. 2010); $1703 v_{\mathrm{t}}=0$ lines up to $J=64$ and $K_{a}=36$ were analyzed together with previous data by means of the rho axis method (RAM; Kleiner 2010). Finally, the experimental dipole moment components of the $\mathrm{DCOOCH}_{3}$ species were measured for the first time, using the Stark spectrometer in Oslo.

We here extend the spectral recording to the $\mathrm{THz}$ region. A new spectral analysis of $3763 v_{\mathrm{t}}=0$ lines up to $1466 \mathrm{GHz}$ (with highest values of $J=69$ and $K_{a}=36$ ), combined with the previous published data, was achieved with the RAM approach. Most of the new data set consists of more than $2000\left|\Delta K_{a}\right|=1$ lines, while in the previous study, about $1100 \Delta K_{a}=0$ lines and $600\left|\Delta K_{a}\right|=1$ lines were analyzed. The highest $K_{a}$ values involved in the $\left|\Delta K_{a}\right|=1$ transitions are 16 in the previous data set, and in the new data set, they are 36 . Hence, our study represents a significant improvement over the former (Margulès et al. 2010). In addition to the increase of data and the considerable extension of the spectral ranges, the analysis is also improved with the RAM torsional-rotational Hamiltonian. This is stated because (i) some transitions from the previous data set were no longer excluded in the analysis; and (ii) the rms for the subsets of lines in which the new data are excluded from the statistics has in general been considerably reduced. The global fit resulted in a unitless standard deviation of $\sigma=0.97$ by varying 27 parameters versus the former results $(\sigma=1.11$ and 24 fitted parameters). The present analysis aims at providing a comprehensive spectral catalog that encompasses as much as possible the frequency coverage of the new-generation far-IR and submillimeter wave observation facilities such as ALMA (84-950 GHz), Herschel (HIFI covers 480-1250 and 1410$1910 \mathrm{GHz})$, and SOFIA $(249 \mathrm{GHz}-10 \mathrm{THz})$. It is expected that these new data represent a useful resource when estimating the deuterium abundance and the physical conditions of the ISM surveys.

The structure of the paper is the following: the experimental details of the recording of the molecular spectra of $\mathrm{DCOOCH}_{3}$ in the laboratory are given in Sect. 2. The analysis of the spectrum and the resulting spectroscopic parameters are presented in Sect. 3, and the conclusions are given in Sect. 4.

\section{Experiments}

The broadband direct multiplication spectrometer at the Jet Propulsion Laboratory (JPL; Drouin et al. 2005, 2013) was employed to collect a complete spectrum from $850-930 \mathrm{GHz}$, 950-1060 GHz, 1060-1205 GHz, 1305-1320 GHz, and 1425$1480 \mathrm{GHz}$. All these spectra were recorded in $2 f$ detection, which resulted in second-derivative line shapes. The recording was performed at room temperature with a 1.2-m-long cell and with $\mathrm{DCOOCH}_{3}$ sample pressure ranging from 30 mTorr to 50 mTorr. The data below $1205 \mathrm{GHz}$ were assigned an uncertainty of $100 \mathrm{kHz}$, the data above $1205 \mathrm{GHz}$ an uncertainty of $200 \mathrm{kHz}$. Note that in these frequency regions, the typically achieved experimental uncertainty is $50-100 \mathrm{kHz}$ for strong isolated lines. The assigned uncertainties in the present work are larger since the spectra are dominated by lines that are neither strong nor isolated.

\section{Spectral analysis}

\subsection{Theoretical model}

We used the so-called RAM internal rotation Hamiltonian in the assignment procedure of the monodeuterated isotopolog of methyl formate $\left(\mathrm{DCOOCH}_{3}\right)$. This formalism is based on the works of Lin \& Swalen (1959), Kirtman (1962), Lees \& Baker (1968) and was originally conceived for the spectral analysis of molecules with one $\mathrm{CH}_{3}$ top undergoing large-amplitude torsion. Later on, this model was extended to include higher order perturbation terms (Herbst et al. 1984; Hougen et al. 1994; Kleiner 2010).

The effective RAM Hamiltonian has been developed to minimize the rotation-torsion interaction by rotating the molecular principal frame system to the so-called rho axis system (Kleiner 2010). This method can be considered as a "global analysis method" because all torsional levels (labeled with the torsional quantum number $v_{\mathrm{t}}$ ), up to a given truncation limit, as well as their $A$ - and $E$-symmetry sublevels are taken into account simultaneously in the Hamiltonian matrix (Kleiner et al. 1996b; Kleiner 2010).

This formalism and the corresponding software (the BELGICs code) have been applied with success to a number of internal rotor molecules observed in the ISM, such as acetaldehyde $\mathrm{CH}_{3} \mathrm{CHO}$ (Kleiner et al. 1996a), acetamide $\mathrm{CH}_{3} \mathrm{CONH}_{2}$ (Hollis et al. 2006), acetic acid $\mathrm{CH}_{3} \mathrm{COOH}$ (Ilyushin et al. 2008, 2013), and different isotopologs of methyl formate (Carvajal et al. 2007, 2009, 2010; Ilyushin et al. 2009; Margulès et al. 2010; Tercero et al. 2012; Haykal et al. 2014). The BELGI code used in this study is available on the web ${ }^{1}$. The Hamiltonian terms, such as the rotational, internal rotation and the coupling terms between

\footnotetext{
1 The source code BELGI for the fit, an example of input data file, and a readme file are available at the web site (http://www. ifpan. edu . $\mathrm{pl} / \mathrm{kisiel/introt/introt.htm \# belgi)} \mathrm{managed} \mathrm{by} \mathrm{Zbigniew}$ Kisiel. Extended versions of code are also available from one of the authors (Kleiner).
} 
Table 1. Root-mean-square (rms) deviations from the global fit ${ }^{a}$ of transitions involving $v_{\mathrm{t}}=0$ torsional energy levels of deuterated methyl formate $\left(\mathrm{DCOOCH}_{3}\right)$.

\begin{tabular}{|c|c|c|c|c|c|c|}
\hline \multicolumn{4}{|c|}{$\begin{array}{l}\text { Number of parameters } \\
\text { Number of lines } \\
\text { rms of the } 3763 v_{\mathrm{t}}=0-0 \text { lines } \\
\text { rms of the } 2232 \mathrm{~A} \text { symmetry lines } \\
\text { rms of the } 1531 \mathrm{E} \text { symmetry lines }\end{array}$} & \multicolumn{3}{|c|}{$\begin{array}{c}27 \\
3763 \\
0.1043 \mathrm{MHz} \\
0.1072 \mathrm{MHz} \\
0.0998 \mathrm{MHz} \\
\end{array}$} \\
\hline Source $^{b}$ & $\operatorname{Range}^{c}(\mathrm{GHz})$ & $J_{\max }, K_{\max }^{d}$ & Number of lines ${ }^{e}$ & Uncertainties $^{f}(\mathrm{MHz})$ & $\mathrm{rms}^{g}(\mathrm{MHz})$ & $\sigma^{h}$ \\
\hline FTMW-Lille & 4-18 & 7,4 & $\begin{array}{r}18 \\
21 \\
\end{array}$ & $\begin{array}{l}0.003 \\
0.010 \\
\end{array}$ & $\begin{array}{l}0.0029 \\
0.0181 \\
\end{array}$ & $\begin{array}{l}0.96 \\
1.81 \\
\end{array}$ \\
\hline BWO-Lille & $580-661$ & 64,36 & 485 & 0.050 & 0.0506 & 1.01 \\
\hline OEST & $91-377$ & 30,25 & 816 & 0.080 & 0.0581 & 0.73 \\
\hline $\begin{array}{l}\text { BWO-Lille } \\
\text { JPL }^{i}\end{array}$ & $\begin{array}{c}580-661 \\
850-1205 \\
\end{array}$ & $\begin{array}{l}57,29 \\
69,36 \\
\end{array}$ & $\begin{array}{c}50 \\
1814 \\
\end{array}$ & $\begin{array}{l}0.100 \\
0.100 \\
\end{array}$ & 0.1041 & 1.04 \\
\hline OSLO & 7-80 & 48,16 & $\begin{array}{c}260 \\
16\end{array}$ & $\begin{array}{l}0.150 \\
0.200\end{array}$ & & \\
\hline $\begin{array}{c}\text { BWO-Lille } \\
\text { CURL } \\
\text { OEST } \\
\text { JPL }^{i}\end{array}$ & $\begin{array}{c}580-661 \\
227-290 \\
91-377 \\
1205-1466\end{array}$ & $\begin{array}{c}57,35 \\
5,1 \\
26,6 \\
69,35\end{array}$ & $\begin{array}{c}31 \\
5 \\
10 \\
237\end{array}$ & $\begin{array}{l}0.200 \\
0.200 \\
0.200 \\
0.200\end{array}$ & 0.1728 & 0.94 \\
\hline
\end{tabular}

Notes. ${ }^{(a)}$ Parameter values are given in Table 2. The complete list of observed minus calculated residuals of $v_{\mathrm{t}}=0$ lines are given in supplementary Table A.1. ${ }^{(b)}$ Sources of data: OEST (Oesterling et al. 1995); BWO-Lille, FTMW-Lille and OSLO data come from Margulès et al. (2010) and CURL data come from (Curl 1959). JPL data have been measured in this work. ${ }^{(c)}$ Spectral range of the measurements. ${ }^{(d)}$ Maximum $J$ and $K_{a}$ for each group of measurements. ${ }^{(e)}$ Number of lines in each uncertainty group. ${ }^{(f)}$ Measurement uncertainty in MHz used in the fit. ${ }^{(g)}$ Root-meansquare deviation in MHz for each group. ${ }^{(h)}$ Unitless standard deviation of each set of measurements (where the differences between the observed and calculated frequencies in the standard deviation formula are weighted with their experimental uncertainties). ${ }^{(i)}$ The spectrometer spectral range for these measurements is $850-1466 \mathrm{GHz}$. The experimental uncertainty is generally better than $0.100 \mathrm{MHz}$ for isolated lines below $1205 \mathrm{GHz}$, but some lines are either blended or present a poor signal-to-noise ratio, therefore their measurement accuracy was taken to be $0.200 \mathrm{MHz}$ according to the broadening of the experimental line. Above $1205 \mathrm{GHz}$, the experimental uncertainty for isolated lines is considered better than $0.200 \mathrm{MHz}$.

the internal rotation and the global rotation, as well as other details for the application of the code to methyl formate species are described in Carvajal et al. (2007).

\subsection{Assignments and fit}

We began our analysis by generating a line prediction using the parameters from Margulès et al. (2010) in the spectral frequency ranges of the JPL spectrometer. Consequently, new assignments were performed based on the comparison between the predicted and experimental lines, the new transitions were fitted progressively from the lowest to highest $J$ values up to a highest value of $J$ and $K_{a}$ of 69 and 36, respectively.

We analyzed the available data for $\mathrm{DCOOCH}_{3}$, which span the following frequency ranges: 4-80, 91-377, 580-661, and 850-1466 GHz. The spectral lines were taken from the new JPL measurements and from those obtained in previous studies (Curl 1959; Oesterling et al. 1995; Margulès et al. 2010). A total of 3763 transition lines for the ground torsional $A$ - and $E$-symmetry substates were fitted with the following experimental uncertainties: (i) the 5 lines from Curl (1959) are weighted with an estimated measurement uncertainty of $200 \mathrm{kHz}$; (ii) the 816 lines and 10 lines from Oesterling et al. (1995) are weighted with uncertainties of 80 and $200 \mathrm{kHz}$, respectively; (iii) of the lines from Margulès et al. (2010), 18 lines are weighted with an uncertainty of $3 \mathrm{kHz}, 21$ with an uncertainty of $10 \mathrm{kHz}, 485$ with an uncertainty of $50 \mathrm{kHz}, 50$ lines with $100 \mathrm{kHz}, 260$ with an uncertainty of $150 \mathrm{kHz}$, and 47 with an uncertainty of $200 \mathrm{kHz}$; and (iv) the 1814 and 237 newly measured lines recorded with the JPL spectrometer (present work) are weighted with uncertainties of 100 and $200 \mathrm{kHz}$. The experimental uncertainty of JPL data is generally better than $100 \mathrm{kHz}$ for isolated lines below $1205 \mathrm{GHz}$, but many lines are either blended or present a poor signal-tonoise ratio. For these latter cases, the measurement accuracy is taken to be $200 \mathrm{kHz}$ according to the broadening of the experimental line. Above $1205 \mathrm{GHz}$, the experimental uncertainty for isolated lines is considered to be better than $200 \mathrm{kHz}$. During the fitting process, we noticed that a few lines (9) from Margulès et al. (2010), which were assigned in that study but were not included in the fit at that time because they did not match, are now well reproduced. Therefore we decided to include in our fit six, two, and one of the previously assigned lines with uncertainties of $50 \mathrm{kHz}, 100 \mathrm{kHz}$, and $150 \mathrm{kHz}$, respectively.

The overall quality of the fit is shown in Table 1, which gives the root-mean-square deviations for transitions according to their measurement uncertainties. In general, each group of transitions fits the experimental uncertainties, and the previous results have improved significantly. A comparison between the rms for the subsets of data without new recorded lines obtained from the analysis of Margulès et al. (2010) and the present work is useful to assess the improvement of this new fit. The rms has changed from 0.0047 to $0.0029 \mathrm{MHz}, 0.0154$ to $0.0181 \mathrm{MHz}$, $0.0655 \mathrm{MHz}$ to $0.0506 \mathrm{MHz}$, and 0.0799 to $0.0581 \mathrm{MHz}$ for the subset of lines with experimental uncertainties of $3 \mathrm{kHz}, 10 \mathrm{kHz}$, $50 \mathrm{kHz}$, and $80 \mathrm{kHz}$, respectively. The global unitless standard deviation of the fit is 0.97 (equivalent to $104.3 \mathrm{kHz}$ of standard deviation), where 2232 lines corresponding to A-symmetry and 1531 corresponding to E-symmetry were fitted with root-meansquare deviations of $107.2 \mathrm{kHz}$ and $99.8 \mathrm{kHz}$, respectively.

The 27 fitted rotation-torsion parameter values (plus the internal rotation parameter $F$ kept fixed, see below) used in our approach are presented in Table 2 . The best fit includes terms up to sixth order, that is, those with $n=l+m=6$, where $n$ is the total order of the operator, $l$ is the order of the torsional part, and 


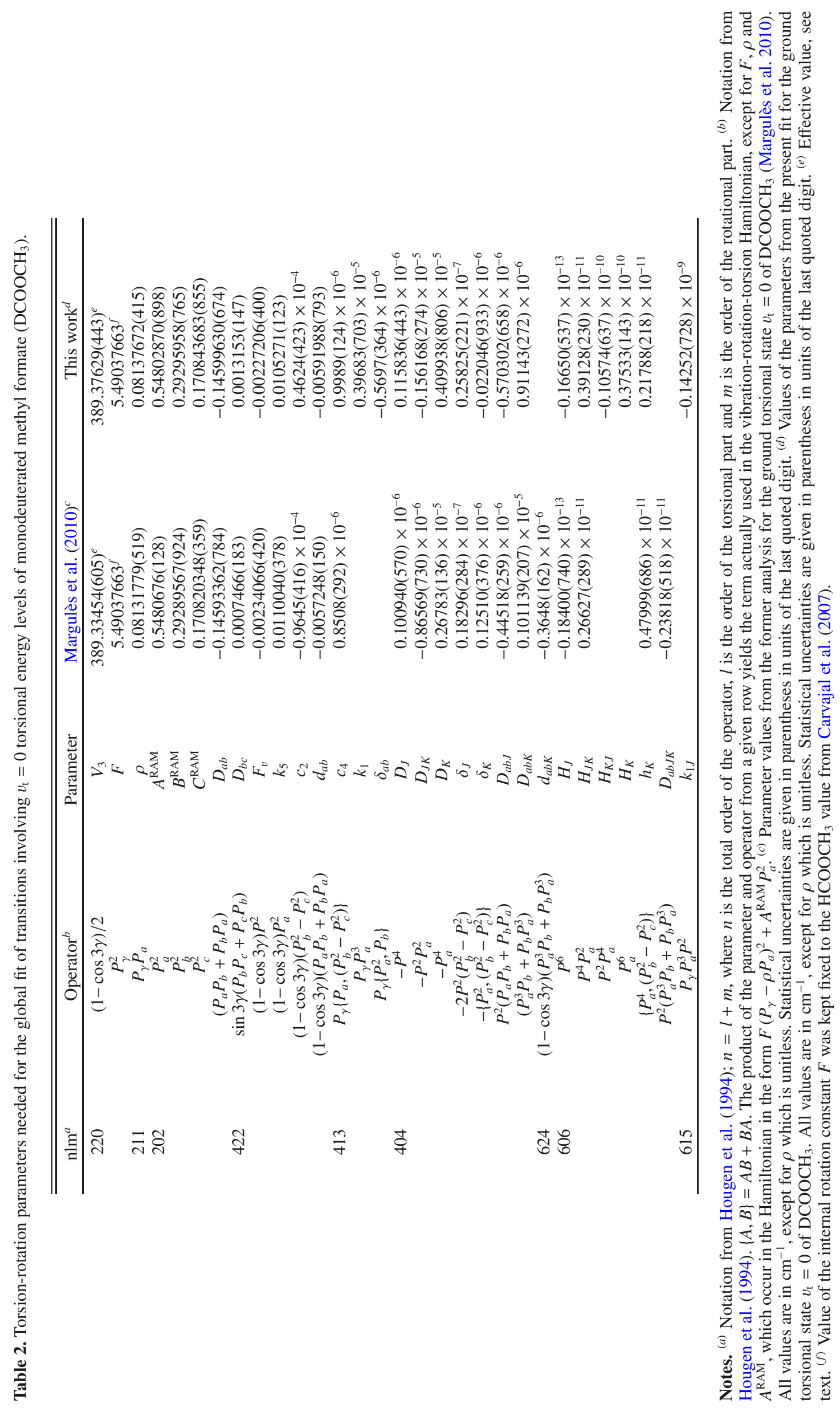


C. Duan et al.: Rotational spectrum of $\mathrm{DCOOCH}_{3}$ methyl formate

Table 3. Rotational constants in the RHO axis system (RAM) and in the principal axis system (PAM) for $\mathrm{DCOOCH}_{3}$.

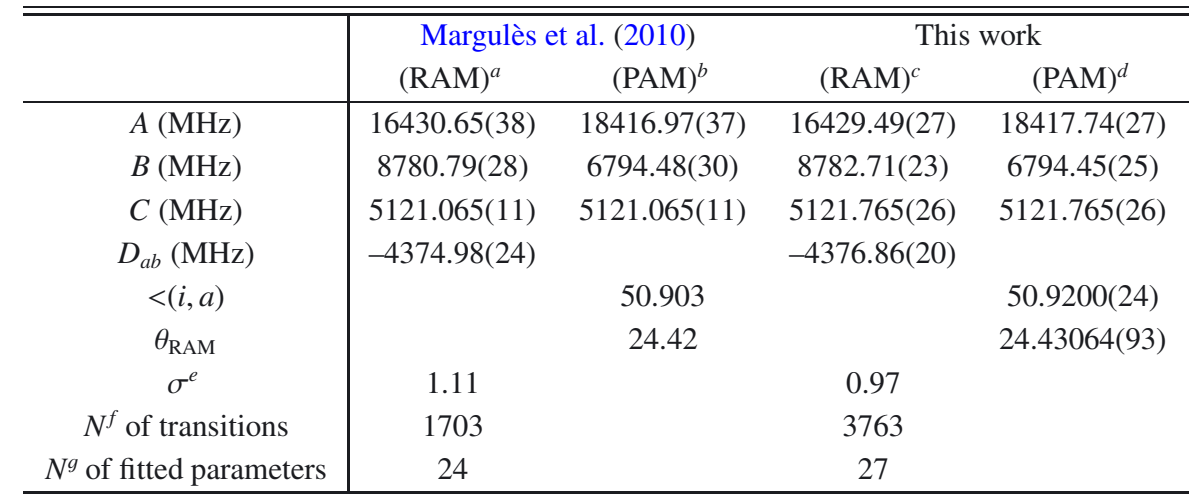

Notes. ${ }^{(a)}$ Rotational constants obtained in the former work for RAM-axis system (Margulès et al. 2010). ${ }^{(b)}$ Rotational constants of the former work transformed to the principal axis system and angles $<(i, a)$ and $\theta_{\mathrm{RAM}}$ in degrees between the principal $a$-axis and the methyl top axis $(i)$ and between the principal $a$-axis and the RAM $a$-axis (Margulès et al. 2010). ${ }^{(c)}$ Rotational constants obtained in this work for the RAM-axis system (see Table 2). ${ }^{(d)}$ Rotational constants of this work transformed to the principal axis system and angles $<(i, a)$ and $\theta_{\text {RAM }}$ in degrees between the principal $a$-axis and the methyl top axis $(i)$ and between the principal $a$-axis and the RAM $a$-axis. ${ }^{(e)}$ Unitless standard deviation. ${ }^{(f)}$ Number of transitions included in the fit. ${ }^{(g)}$ Number of fitted Hamiltonian parameters.

$m$ is the order of the rotational part, respectively, following the notation of previous papers on the RAM method (Hougen et al. 1994). In comparison with the previous work (Margulès et al. 2010), we only need three more parameters for 2060 new lines to achieve nearly experimental accuracy. We note that the values of the parameters determined in the present work have, in general, higher absolute values and smaller uncertainties than those derived in the previous work. The number of experimental lines for the spectral analysis has roughly doubled, therefore, substantial changes in the parameter values are observed with variations three times their uncertainties. Moreover, we highlight that some parameters such as the centrifugal constants have different values. All this is explained because new lines and different fitted parameters were used in the spectral analysis, for example, the $D_{a b}$ value is affected by the new fit of the $\delta_{a b}$ parameter and the elimination in the model of $d_{a b K}$ and $D_{a b J K}$ with respect to the former analysis, and the quartic centrifugal constant values have changed by the fit of new sextic rotational parameters.

We kept the internal rotation constant $F$ fixed because no direct information on the first torsional $\left(v_{t}=1\right)$ band is included in the fit. Indeed, in this situation, there is a strong correlation between $F$ and the potential barrier height $V_{3}$ (Carvajal et al. 2009, 2010). For the same reason, the value of the potential parameter $V_{6}$ could not be determined. The internal rotation parameter $F$ was fixed to the experimental value for $\mathrm{H}^{12} \mathrm{COO}^{12} \mathrm{CH}_{3}$ derived by Carvajal et al. (2007) and $V_{6}$ was kept fixed to zero. Hence, the value of $V_{3}$ determined in our fit can only be considered as an effective value containing the contribution of $V_{6}$, as was highlighted in Carvajal et al. (2009).

Table A.1 only contains a part of the entire table of the fitted lines of $\mathrm{DCOOCH}_{3}$. It provides the line assignments, the observed frequencies with the experimental uncertainties in parentheses, the computed frequencies with their uncertainties in parentheses, the observed-to-calculated differences, the line strengths for the transitions in the ground state, the lower state energies relative to the A-symmetry $J=K_{a}=0$ level taken as the zero of energy, and the references of the data sources. Blended lines are marked in the table, and some of them show observed-to-calculated values with high residuals.
The transformation of the RAM rotational constants into the principal axis system (PAM) values is given in Table 3 according to the procedure described in Carvajal et al. (2007). The RAM rotational constants obtained in the fit (see Table 2) and their transformed PAM values are given in $\mathrm{MHz}$ in addition to the rotation angle $\theta_{\text {RAM }}$ about the out-of-plane $c$-axis of the RAM axis system into PAM axis system. The variation of the angle $<(i, a)$ between the $\mathrm{C}_{3}$ symmetry axis of the methyl-top and the $a$-principal axis is also provided. This table is useful for two reasons: (i) to compare the parameter values between different analysis methods; and (ii) to transform the dipole moment components that are experimentally obtained in the PAM into the rho frame axis system of the molecular species under study. We also compare our fit and that of the former analysis (Margulès et al. 2010). The parameter values of $B^{\mathrm{RAM}}$ and $D_{a b}$ vary by about $1.9 \mathrm{MHz}$ with respect to the previous work. These changes are caused by the significant increase in the number of fitted experimental lines and the fit of different parameters in the Hamiltonian model that could present a relatively strong correlation, that is, $D_{a b}$ is fitted with $\delta_{a b}$ in our analysis and was fitted with $d_{a b K}$ and $D_{a b J K}$ previously. Nevertheless, the rotational transformation of the rho axes into the principal axes provides similar values for $B^{\mathrm{PAM}}$.

\subsection{Intensity calculations}

The intensity calculations for $\mathrm{DCOOCH}_{3}$ were performed through the procedure described for the main isotopolog of methyl formate (Carvajal et al. 2007).

The experimental dipole moment components for this species were measured in Margulès et al. (2010). Their principal axis system values were $\mu_{a}=1.643(12) \mathrm{D}, \mu_{b}=0.748(11) \mathrm{D}$ and $\mu_{c}=0.0 \mathrm{D}$ (assumed). The RAM dipole moment components were computed by using the angle $\theta_{\mathrm{RAM}}=24.4306^{\circ}$ of Table 3 in Eq. (2) of Carvajal et al. (2007). The RAM dipole moment components are 1.8053 and $0.0015 \mathrm{D}$ for $\mu_{a}$ and $\mu_{b}$, respectively. The line strengths for the assigned transitions are presented in the supplementary Table A.1.

A portion of the experimental and simulated spectrum in the frequency range $1138.450-1138.570 \mathrm{GHz}$ is presented in Fig. 1. 


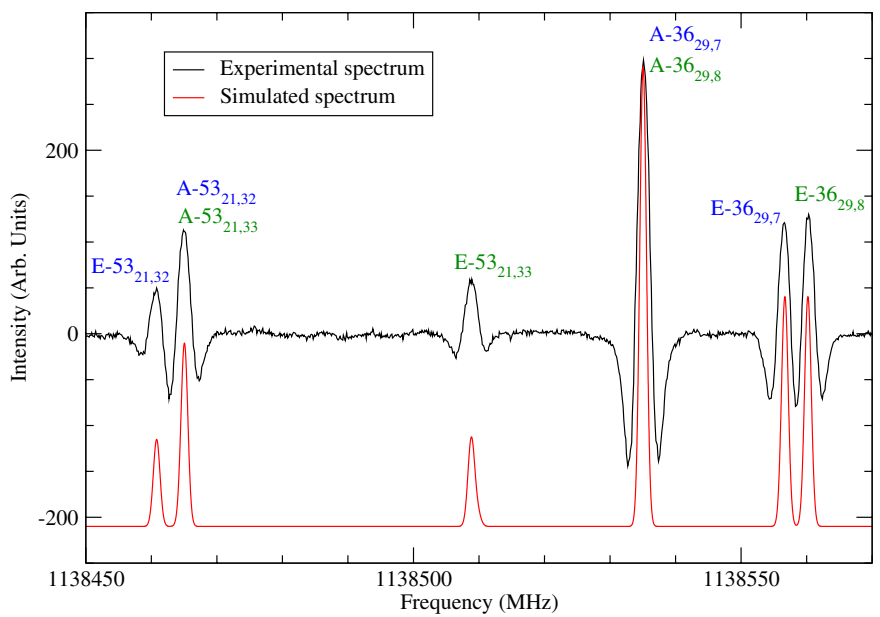

Fig. 1. Portion of the experimental spectrum recorded with the JPL experimental setup in the frequency range 1138 450-1 $138570 \mathrm{MHz}$. The simulated spectrum is also provided by using the Hamiltonian parameter values given in Table 2 and the dipole moment components as explained in the text. The involved features are assigned as $\Gamma-J_{K_{a}, K_{c}}$ for a transition to a state with $J^{\prime}=J-1, K_{a}^{\prime}=K_{a}-1$, and $K_{c}^{\prime}=K_{c}$ and with symmetry $\Gamma$ (A or E). Transitions $J=K_{a}+K_{c}$ are labeled in blue and $J+1=K_{a}+K_{c}$ in green.

This allows us to provide a first assessment of the agreement between the experimental and computed frequencies and line strengths. The involved features are assigned as $\Gamma-J_{K_{a}, K_{c}}$ for a transition to a state with $J^{\prime}=J-1, K_{a}^{\prime}=K_{a}-1$, and $K_{c}^{\prime}=K_{c}$ and with symmetry $\Gamma$ (A or E). Transitions $J=K_{a}+K_{c}$ are labeled in blue and $J+1=K_{a}+K_{c}$ in green.

\section{Conclusions}

We newly measured the rotational spectrum of $\mathrm{DCOOCH}_{3}$ in the JPL laboratory in the frequency range from 0.85 to $1.5 \mathrm{THz}$. We assigned 2060 new features and fitted them jointly with the previously recorded data. In total, 3763 transitions in the ground state were analyzed with a unitless standard deviation of 0.97 by using the rho axis method. The fitted parameters were provided and compared with previous work. The accuracy of our parameter values is higher in general, and the new global result is significantly better. This is shown by comparing the rms of the subsets of data, in which the new lines are excluded, in the previous and our analysis. We presented the resulting list of all available assigned lines with their experimental and calculated frequencies, line strengths, and lower state energies. This study aims at completing the line list for this species and also intends to encompass the frequency coverage of the new-generation far-IR and submillimeter wave observation facilities. This work could be relevant in estimating accurate interstellar $\mathrm{D} / \mathrm{H}$ ratios that will provide clues of the formation history of methyl formate. Our line list may help to resolve anomalous $\mathrm{D} / \mathrm{H}$ ratios observed and to decipher what they means for chemistry and cloud history.

Acknowledgements. This research was supported by the FIS2011-28738-C0202 project (MINECO, Spain), the French PCMI (Programme National de Physique Chimie du Milieu Interstellaire), and the National Natural Science Foundation of China (Grant No. 11174098). M.C. acknowledges the research stay at the Université Paris Diderot under the Guest Faculty programme in May 2014. Portions of this paper present research carried out at the Jet Propulsion Laboratory, California Institute of Technology, under contract with the National Aeronautics and Space Administration. Government sponsorship is acknowledged.

\section{Appendix A: Part of the supplementary tables available at the CDS}

Table A.1. Assignments, observed frequencies, calculated frequencies from the RAM fit, residuals, line strengths, and lower state energy levels for methyl formate isotopologue $\mathrm{DCOOCH}_{3}$ transitions from $v_{\mathrm{t}}=0$ torsional states included in the fit with parameters of Table 2 .

\begin{tabular}{|c|c|c|c|c|c|c|c|c|c|c|c|c|c|c|c|}
\hline \multicolumn{6}{|c|}{ Upper State $^{a}$} & \multicolumn{4}{|c|}{ Lower State $^{a}$} & \multirow[b]{2}{*}{ Obs.Freq.(Unc.) $)^{b}$} & \multirow[b]{2}{*}{ Calc. Freq (Unc.) $)^{c}$} & \multirow[b]{2}{*}{ Obs.-Calc ${ }^{d}$} & \multirow[b]{2}{*}{$S(\mathrm{i} \rightarrow \mathrm{f})^{e}$} & \multirow[b]{2}{*}{ Lower Energy $^{f}$} & \multirow[b]{2}{*}{ Ref. $^{g}$} \\
\hline$v_{\mathrm{t}}^{\prime}$ & $J^{\prime}$ & $K_{a}^{\prime}$ & $K_{c}^{\prime}$ & $p^{\prime}$ & $v_{t}^{\prime \prime}$ & $J^{\prime \prime}$ & $K_{a}^{\prime \prime}$ & $K_{c}^{\prime \prime}$ & $p^{\prime \prime}$ & & & & & & \\
\hline 0 & 55 & 16 & 40 & - & 0 & 54 & 15 & 39 & - & $1019915.551(100)$ & $1019915.479(12)$ & 0.071 & 8.424 & 691.6845 & JPL \\
\hline 0 & 56 & 16 & 41 & - & 0 & 55 & 15 & 40 & - & $1028524.736(100)$ & 1028524.731(13) & 0.006 & 8.312 & 714.1913 & JPL \\
\hline 0 & 57 & 16 & 42 & - & 0 & 56 & 15 & 41 & - & $1036404.097(100)$ & $1036404.175(14)$ & -0.078 & 8.185 & 737.1532 & JPL \\
\hline 0 & 58 & 16 & 43 & - & 0 & 57 & 15 & 42 & - & $1043311.369(100)$ & $1043311.237(16)$ & 0.132 & 8.042 & 760.5795 & JPL \\
\hline 0 & 59 & 16 & 44 & - & 0 & 58 & 15 & 43 & - & $1048936.120(100)$ & $1048935.908(18)$ & 0.212 & 7.882 & 784.4812 & JPL \\
\hline 0 & 60 & 16 & 45 & - & 0 & 59 & 15 & 44 & - & $1052904.968(100)$ & $1052905.217(20)$ & -0.249 & 7.705 & 808.8711 & JPL \\
\hline 0 & 61 & 16 & 46 & - & 0 & 60 & 15 & 45 & - & $4810.666(100)$ & $4810.532(24)$ & & & 833.7 & JPL \\
\hline 0 & 62 & 16 & 47 & - & 0 & 61 & 15 & 46 & - & $264.640(100)$ & $4264.441(29)$ & 0.199 & 319 & 859. & JPL \\
\hline 0 & 63 & 16 & 48 & - & 0 & 62 & 15 & 47 & - & $1050985.044(100)$ & $1050985.011(36)$ & 0.033 & 7.133 & 885.0 & JPL \\
\hline 0 & 64 & 16 & 49 & - & 0 & 63 & 15 & 48 & - & $1044884.792(100)$ & 104488 & -0.226 & 6.978 & & JPL \\
\hline 0 & 67 & 16 & 52 & - & 0 & 66 & 15 & 51 & - & $1012569.476(100)$ & $1012569.535(77)$ & -0.059 & 6.955 & 993.8515 & JPL \\
\hline 0 & 68 & 16 & 53 & - & 0 & 67 & 15 & 52 & - & $999163.977(100)$ & $999163.731(91)$ & 0.246 & 7.166 & 1022.1892 & JPL \\
\hline 0 & 55 & 16 & 40 & & 0 & 54 & -15 & 39 & & $1019903.327(100)$ & 1019903.037(31) & 0.290 & 6.982 & 691.6851 & JPL B \\
\hline 0 & 56 & 16 & 41 & & 0 & 55 & -15 & 40 & & $1028543.113(100)$ & $1028543.040(21)$ & 0.073 & 7.686 & 714.1916 & JPL \\
\hline 0 & 57 & 16 & 42 & & 0 & 56 & -15 & 41 & & $1036444.463(100)$ & $1036444.250(15)$ & 0.213 & 7.939 & 737.1533 & JPL \\
\hline 0 & 58 & 16 & 43 & & 0 & 57 & -15 & 42 & & $1043366.185(100)$ & $1043366.334(15)$ & -0.148 & 7.947 & 760.5793 & JPL \\
\hline 0 & 59 & 16 & 44 & & 0 & 58 & -15 & 43 & & $1049001.868(100)$ & $1049001.726(18)$ & 0.143 & 7.845 & 784.4807 & JPL \\
\hline 0 & 60 & 16 & 45 & & 0 & 59 & -15 & 44 & & $1052979.330(100)$ & $1052979.084(23)$ & 0.246 & 7.689 & 808.8705 & JPL \\
\hline 0 & 61 & 16 & 46 & & 0 & 60 & -15 & 45 & & $1054890.696(100)$ & $1054890.678(31)$ & 0.018 & 7.507 & 833.7619 & JPL \\
\hline 0 & 63 & 16 & 48 & & 0 & 62 & -15 & 47 & & $1051073.005(100)$ & $1051072.806(70)$ & 0.199 & & 885.0925 & JPL \\
\hline 0 & 67 & 16 & 52 & & 0 & 66 & -15 & 51 & & $1012634.611(100)$ & $1012634.884(121)$ & -0.274 & 6.950 & 993.8521 & JPL \\
\hline
\end{tabular}

Notes. ${ }^{(a)}$ Upper and lower state quantum numbers are indicated by ' and " respectively. Torsion-rotation levels of A species have a "parity" label; levels of E species have a signed $K_{a}$ value (Herbst et al. 1984). ${ }^{(b)}$ Observed $v_{\mathrm{t}}=0$ transitions in MHz, with estimated uncertainties in parentheses (in $\mathrm{kHz}$ ). ${ }^{(c)}$ Calculated line frequency in $\mathrm{MHz}$ with calculated uncertainty in $\mathrm{kHz} .{ }^{(d)}$ Differences among the experimental and computed frequencies. ${ }^{(e)}$ Calculated line strengths in $\mathrm{D}^{2}$ (for details of the calculation procedure, see the text). ${ }^{(f)}$ Lower state energy $\left(\mathrm{cm}^{-1}\right)$ referred to the $J=K_{a}=0$ A-species energy level taken as the zero of the energy (zero-point torsional energy: $66.0967 \mathrm{~cm}^{-1}$ ). ${ }^{(g)}$ Sources of data are explained in footnote $\mathrm{b}$ of Table 1 . Blended lines are indicated with a capital letter B. 


\section{References}

Bauder, A. 1979, J. Phys. Chem. Ref. Data, 8, 583

Brown, R. D., Crofts, J. G., Gardner, F. F., et al. 1975, ApJ, 197, L29

Carvajal, M., Willaert, F., Demaison, J., \& Kleiner, I. 2007, J. Mol. Spectr., 246, 158

Carvajal, M., Margulès, L., Tercero, B., et al. 2009, A\&A, 500, 1109

Carvajal, M., Kleiner, I., \& Demaison. J. 2010, ApJS, 190, 315

Churchwell, E., \& Winnewisser, G. 1975, A\&A, 45, 229

Coudert, L. H., Margulès, L., Huet, T. R., et al. 2012, A\&A, 543, A46

Coudert, L. H., Drouin, B. J., Tercero, B., et al. 2013, ApJ, 779, 119

Curl, R. F. 1959, J. Chem. Phys., 30, 1529

Demaison, J., Boucher, D., Dubrulle, A., \& Van Eijck, B. P. 1983, J. Mol. Spectr. 102,260

Demyk, K., Wlodarczak, G., \& Carvajal, M. 2008, A\&A, 489, 589

Drouin, B. J., Maiwald, F. W., \& Pearson, J. C. 2005, Rev. Sci. Instrum., 76, 093113

Drouin, B. J., Pearson, J. C., Yu, S., \& Gupta, H. 2013, IEEE Trans. Terahertz Science and Technology, 3, 314

Favre, C., Despois, D., Brouillet, N., et al. 2011, A\&A, 532, A32

Favre, C., Carvajal, M., Field, D., et al. 2014, ApJS, 215, 25

Haykal, I., Carvajal, M., Tercero, B., et al. 2014, A\&A, 568, A58

Herbst, E., Messer, J. K., De Lucia, F. C., \& Helminger, P. 1984, J. Mol. Spectr., 108,42

Hollis, J. M., Lovas, F. J., Remijan, A. J., et al. 2006, ApJ, 643, L25

Horn, A., Møllendal, H., Sekiguchi, O., et al. 2004, ApJ, 611, 605

Hougen, J. T., Kleiner, I., \& Godefroid, M. 1994, J. Mol. Spectr., 163, 559

Ilyushin, V., Kleiner, I., \& Lovas, F. J. 2008, J. Phys. Chem. Ref. Data, 37, 97

Ilyushin, V., Kryvda, A., \& Alekseev, E. 2009, J. Mol. Spectr., 255, 32

Ilyushin, V., Endres, C. P., Lewen, F., Schlemmer, S., \& Drouin, B. J. 2013, J.

Mol. Spectr., 290, 31

Karakawa, Y., Oka, K., Odashima, H., Takagi, K., \& Tsunekawa, S. 2001, J. Mol. Spectr., 210, 196
Kirtman, B. 1962, J. Chem. Phys., 37, 2516

Kleiner, I. 2010, J. Mol. Spectr., 260, 1

Kleiner, I., Lovas, F. J., \& Godefroid, M. 1996a, J. Phys. Chem. Ref. Data, 25, 1113

Kleiner, I., Hougen, J. T., Grabow, J. U., et al. 1996b, J. Mol. Spectr., 179, 41

Kobayashi, K., Ogata, K., Tsunekawa, S., \& Takano, S. 2007, ApJ, 657, L17

Kobayashi, K., Takamura, K., Sakai, Y., et al. 2013, ApJS, 205, 1

Lees, R. M., \& Baker, J. G. 1968, J. Chem. Phys., 48, 5299

Lin, C. C., \& Swalen, J. D. 1959, Rev. Mod. Phys., 31, 841

Maeda, A., Medvedev, I.R., De Lucia, F. C., \& Herbst, E. 2008a, ApJS, 175, 138

Maeda, A., De Lucia, F. C., \& Herbst, E. 2008b, J. Mol. Spectr., 251, 293

Margulès, L., Coudert, L. H., Møllendal, H., et al. 2009, J. Mol. Spectr., 254, 55

Margulès, L., Huet, T. R., Demaison, J., et al. 2010, ApJ, 714, 1120

Oesterling, L. C., Ferguson, D. W., Herbst, E., \& De Lucia, F. C. 1995, J. Mol. Spectr. 172,469

Oesterling, L. C., Albert, S., De Lucia, F. C., Sastry, K. V. L. N., \& Herbst, E. 1999, ApJ, 521, 255

Ogata, K., Odashima, H., Takagi, K., \& Tsunekawa, S. 2004, J. Mol. Spectr., 225,14

Plummer, G. M., Blake, G. A., Herbst, E., \& De Lucia, F. C. 1984, ApJS, 55, 633

Plummer, G. M., Herbst, E., De Lucia, F. C., \& Blake, G. A. 1986, ApJS, 60, 949

Shaw, A. M. 2006, Astrochemistry (England: John Wiley \& Sons, Ltd.)

Takano, S., Sakai, Y., Kakimoto, S., Sasaki, M., \& Kobayashi, K. 2012, PASJ, 64,89

Taquet, V., Charnley, S. B., \& Sipilä, O. 2014, ApJ, 791, 1

Tercero, B., Margulès, L., Carvajal, M., et al. 2012, A\&A, 538, A119

Tudorie, M., Ilyushin, V., Vander Auwera, J., et al. 2012, J. Chem. Phys., 137, 064304

Willaert, F., Møllendal, H., Alekseev, E., et al. 2006, J. Mol. Struct., 795, 4 